\title{
Intraoral Swelling in a Neonate: Airway Management a Challenge
}

\author{
Veena Asthana ${ }^{1^{*}}$, Santosh Singh ${ }^{2}$, Abhishek Deshwal $^{1}$ and Turab Singh ${ }^{1}$ \\ ${ }^{1}$ Department of Anesthesiology, Himalayan Institute of Medical Sciences, Dehradun, Uttrakhand, India \\ ${ }^{2}$ Department of Surgery, Himalayan Institute of Medical Sciences, Dehradun, Uttrakhand, India
}

*Corresponding author: Veena Asthana, Department of Anaesthesia, Himalayan Institute of Medical Sciences, Swami Ram Nagar, Dehradun, India, Tel: 09719529195; E-mail: drvasthana@yahoo.co.in

Rec date: Dec 14, 2016; Acc date: Jan 19, 2017; Pub date: Jan 27, 2017

Copyright: (c) 2017 Asthana V et al. This is an open-access article distributed under the terms of the Creative Commons Attribution License, which permits unrestricted use, distribution, and reproduction in any medium, provided the original author and source are credited.

\begin{abstract}
Foregut duplications occurring in tongue have very few reports in literature. These oral and esophageal lesions may cause feeding and respiratory difficulties. For surgical excision securing airway in these babies is very challenging, as visualization of glottis is hampered, because of lack of space in the mouth. We report a case of twelve hours old neonate referred to our institute for the excision of intraoral swelling under general anaesthesia which was revealed as gastric duplication cyst histopathologically.
\end{abstract}

Keywords: Foregut; Airway management; Esophageal lesions

\section{Introduction}

First published by Duncan and Daniel in 1942, foregut duplications occurring in tongue are quite rare, with very few cases reported in literature [1]. Symptoms are often related to the location of the duplication. Oral and esophageal lesions may cause respiratory difficulties .Complete surgical excision is the definitive treatment and should not be delayed, as respiratory compromise is imminent [2]. Orotracheal intubation in these babies is difficult, as retraction of tongue and visualization of glottis is hampered, because of lack of space in the oral cavity and hence, the need for naso-tracheal intubation or tracheostomy may arise [3]. We report a case of twelve hours old neonate referred to our institute for the excision of tongue mass under general anaesthesia.

\section{Case Report}

A twelve hours old neonate, weighing $2.5 \mathrm{~kg}$ with a swelling on a tongue interfering the feeding and closing the mouth was brought to our institute. Vitals of the neonate were stable .Examination revealed that the neonate's mouth was wide opened due to large cystic, non transilluminant mass occupying the entire oral cavity. The origin of the mass could not be defined and it was occupying the entire ventral aspect of the tongue (Figure 1). In the operation theater the monitors were attached.

Due to unavailability of paediatric fiberoptic bronchoscope in our set up, preparation for securing surgical airway in case of failed intubation was kept ready by us. We ensured the ease of bag and mask ventilation and then to attempt endotracheal intubation using inhalational anaesthetics with preservation of the spontaneous breaths. Anaesthesia was induced with $1 \%$ increments of sevoflurane, up to $6 \%$ in $100 \%$ oxygen. While maintaining spontaneous ventilation, injection atropine $0.01 \mathrm{mg} / \mathrm{kg}$ and injection fentanyl 2 microgram $/ \mathrm{kg}$ was administered. On attaining adequate depth of anaesthesia, direct laryngoscopy was carried out using the paraglossal approach with a size 0 Miller's blade additionally the view improved to some extent with optimal external laryngeal manipulation (Figure 2).

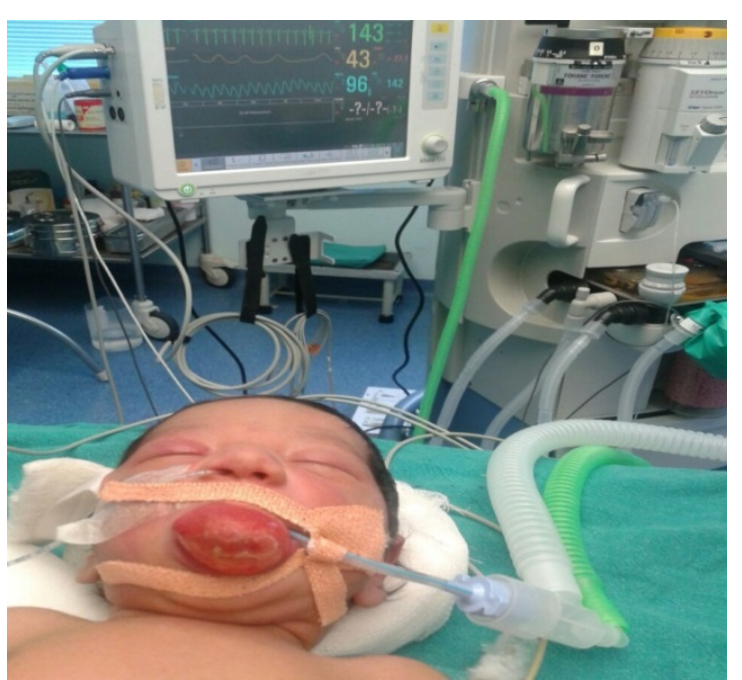

Figure 1: Swelling of the tongue in the neonate.

A $3.5 \mathrm{~mm}$ internal diameter endotracheal tube loaded with a stylet was placed in the trachea. After confirmation of tube placement, it was fixed at the corner of the mouth and atracurium $1.5 \mathrm{mg}$ administered intravenously. Anaesthesia was maintained by intermittent positive pressure ventilation with oxygen, nitrous oxide and sevoflurane (1\%). The intraoperative period was uneventful and the patient was extubated after surgery.

\section{Discussion}

Gastro-intestinal duplication cyst can arise anywhere from oral cavity to anus. Chen et al. [3] reported two cases of sublingual duplication cysts and concluded that intraoral cyst as a large sublingual mass is rare and has the potential for airway obstruction and respiratory distress at delivery which may necessitate immediate tracheostomy. Fiberoptic intubation remains the "gold standard" for 
establishing tracheal intubation in the difficult paediatric airway despite its limitations.

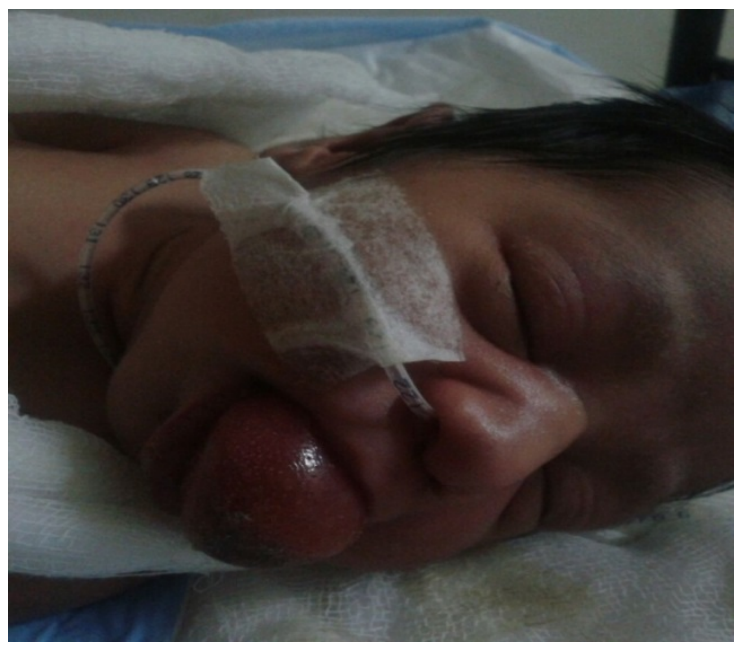

Figure 2: Airway management in neonate.

Securing airway in these paedtric patients is challenging task for the anaesthesiologist due to limited space in the oral cavity along with obscured view of the glottis resulting the need for naso-tracheal intubation or tracheostomy [3]. Although there may be need for aspiration to decompress the cyst and achieve relief from symptoms, this alternate seems a temporary emergency measure because of the risk of infection [4].

As we anticipated difficult intubation we focused on the difficult airway algorithm preserving the spontaneous ventilation using inhalational induction. Sevoflurane was preferred due to its low blood gas solubility, least irritating effect on the airways along with maintainence of depth of anaesthesia that can be rapidly altered and the patient can be awakened if optimum airway control is not achieved [5].

The success in the glottic visualization using different blades has been explained by Racz [6]. We used Miller blade size 0 using paraglossal approach. Paraglossal straight blade laryngoscopy may be helpful over use of the Macintosh technique when intubation is unexpectedly difficult [7]. The straight blade overcomes the problem of interference of Macintosh blade curvature into the line of sight. Secondly the improved glottis view by head extension is possible with use of straight blade but not with curved blade [8]. Similarly others have tried the molar approach also a variant of paraglossal technique [9]. Several methods of optimizing the view with this technique have been advocated $[10,11]$. Miller blade is passed from the right corner of the mouth. It is inserted along the groove between the tongue and the tonsil, using leftward and anterior pressure to displace the tongue to the left of the laryngoscope and to maintain the tongue in this position at all times, the epiglottis identified and the tip of the blade is passed posterior to the epiglottis. The blade is lifted anteriorly, elevating the epiglottis directly so that the glottis is exposed. Studies have demonstrated that external laryngeal manipulation improved glottic visualization [12] which definitely helped us in glottis visualization. As there was not enough space to introduce the endotracheal tube due to the mass hence an assistant was asked to pull the right side of the angle of the mouth to make room to manipulate the endotracheal tube.

\section{Conclusion}

Paraglossal approach can be used as an alternative intubation technique in patients with intraoral swellings where the conventional method fails.

\section{References}

1. El-Bitar MA, Milmoe G, Kumar S (2003) Intralingual foregut duplication cyst in a newborn. Ear Nose Throat J 82: 454-463.

2. Ameh EA, Jimoh AO, Rafindadi AH, Shehu SM (2000) Sublingual gastric duplication cyst causing respiratory obstruction: Case report. East Afr Med J 77: 394-395.

3. Chen MK, Gross E, Lobe TE (1997) Perinatal management of enteric duplication cysts of the tongue. Am J Perinatol 14: 161-163.

4. Barnes L (2001) Surgical pathology of the head and neck 2nd ed. New York: Marcel Dekker 1481-1485.

5. Henderson JJ (1997) The use of paraglossal straight blade laryngoscopy in difficult tracheal intubation. Anaesthesia 52: 552-560.

6. Péan D, Floch H, Beliard C, Piot B, Testa S, et al. (2010) Propofol versus sevoflurane for fiberoptic intubation under spontaneous breathing anesthesia in patients difficult to intubate. Minerva Anestesiol 76: 780-786.

7. Racz GB (1984) Improved vision modification of the Macintosh laryngoscope. Anaesthesia 39: 1249-1250.

8. Agrawal S, Asthana V, Meher R, Singh DK (2008) Paraglossal straight blade intubation technique-an old technique revisited in difficult intubations: A series of 5 cases. Indian J Anaesthesia 52: 317-320.

9. Mittal A, Dwivedi Y, Joshi K, Saxena A, Gupta A (2011) Molar approach of intubation in aneonate with large intraoral swelling. Indian J Anaesth 25-30.

10. McIntyre JWR (1987) The difficult tracheal intubation. Can J Anaesth 34: 204-213.

11. Krantz MA, Poulos JG, Chaouki K, Adamck P (1993) The laryngeal lift: a method to facilitate endotracheal intubation. J ClinAnesth 5: 279-301.

12. Benumof JL, Cooper SD (1996) Quantitative improvement in laryngoscopic view by optimal external laryngeal manipulation. J Clin Anesth 8: 136-140. 